\section{Präventionsprogramm Haut für das Back- sowie Hotel- und Gaststättengewerbe}

\author{
Neue Wege in der Prävention von \\ Berufsdermatosen
}

\author{
A. Bauer ${ }^{1}$, D. Kelterer ${ }^{1}$, M. Stadeler ${ }^{2}$, W. Schneider ${ }^{2}$, \\ P. Kleesz ${ }^{2}$, P. Elsner ${ }^{1}$ \\ ${ }^{1}$ Klinik für Hautkrankheiten der Friedrich-Schiller-Universität Jena \\ (Direktor: Prof. Dr. P. Elsner) \\ ${ }^{2}$ Geschäftsbereich Prävention-Gesundheitsschutz der Berufsge- \\ nossenschaft Nahrungsmittel und Gaststätten Erfurt/Mannheim
}

Zusammenfassung. Unter den Verdachtsmeldungen zur Berufskrankheit (BK) machen die Hauterkrankungen einen großen Anteil aus. Das betrifft neben anderen Gewerben auch das Back-, Hotel- und Gaststättengewerbe. Um die Betreuung der Versicherten zu verbessern, wurde 1999 das „Präventionsprogramm Haut für das Back-, sowie Hotel und Gaststättengewerbe“ von der Abteilung für Allergologie und Berufsdermatologie der Klinik für Hautkrankheiten der Friedrich-Schiller-Universität in Jena und dem arbeitsmedizinischen Präventionszentrum der Berufsgenossenschaft für Nahrungsmittel und Gaststätten (BGN) in Erfurt initiiert, in das bisher 23 Versicherte aufgenommen wurden. Nach einer detaillierten Expositionsanalyse am Arbeitsplatz sowie berufsdermatologischer Anamnese und Untersuchung werden bisherige Diagnosen und Therapiestrategien reevaluiert und falls notwendig ergänzt. Individuelle Hautschutz-, -reinigungs und -pflegeprogramme werden erarbeitet, dem Versicherten erklärt und mitgegeben. Durch die BGN werden die dazu nötigen Mittel zur Verfügung gestellt, Hautschutzseminare und Nachuntersuchungen initiiert. Seit Januar 1999 wurden 16 Versicherte in der berufsdermatologischen Sprechstunde der Klinik für Hautkrankheiten vorstellig. Die bisherigen Ergebnisse zeigen ein Defizit in der Aufklärung der Arbeitnehmer über die Entstehungsursachen und Verhinderungsmöglichkeiten berufsbedingter Hauterkrankungen sowie in der Bereitstellung und Anwendung adäquater Hautschutz-, Hautreinigungs- und -pflegemittel. Bei fast allen Versicherten (75\%) lag ein subtoxisch-kumulatives Handekzem als Erstdiagnose vor. In 7 Fällen (44\%) ist derzeit eine Verlaufsanalyse möglich. Bei 3 Versicherten (43\%) ist es im Beobachtungszeitraum zu einer Besserung bzw. Abheilung der Hautveränderungen gekommen. Bei weiteren 3 Versicherten (43\%) blieb der Hautbefund trotz Verbleib im Beruf stabil. Diese ersten Ergebnisse sind erfolgversprechend. Es ist geplant weitere Arbeitnehmer einzubeziehen und das Programm auf mehrere Zentren auszuweiten.

Prevention of Occupational Skin Hand Dermatitis in Bakers, Confectioners and Employees in Catering Trades. Occupational skin diseases lead the statistical reports of occupational disease (OD). Besides other professions the bakers, confectioners and the employees in the catering trades are at high risk

Akt Dermatol 2001; 27: 219-222

(c) Georg Thieme Verlag Stuttgart · New York ISSN 0340-2541 to develop occupational skin diseases (OSD). Since January 1999 23 employees were included in an interdisciplinary skin disease prevention program run by the Department of Dermatology, Friedrich-Schiller-University of Jena, the Research Center for Applied System Safety and Industrial Medicine, Erfurt and the Technical Experts of the Social Insurance for the Food Industry and Related Professions (Berufsgenossenschaft für Nahrungsmittel und Gaststätten [BGN]), Erfurt. After an in-detail analysis of the occupational exposure of the employees and the personal occupational disease history, diagnosis and therapy were re-evaluated and supplemented, if necessary. Individual skin care and protection regimes will be demonstrated and practised. Skin care and protection ointments as well as protective gloves were supplied by the BGN. Skin care and protection seminars were organised. Since January 199916 employees were examined in the occupational clinic. $75 \%$ of the employees had an irritant contact dermatitis. The preliminary results showed that most of the occupational skin diseases are due to no, wrong or misplaced skin care and protection habits. The follow-up data of 7 (44\%) employees were shown. In the first few month $43 \%$ of the employees improved and/or the skin disease healed. Moreover, in $43 \%$ of the employees the skin condition was stable although they stayed at work. These first results are promising. More employees will be included and the program will be expanded.

\section{Einleitung}

Berufsbedingte Hauterkrankungen bei Arbeitnehmern in der Nahrungsmittelherstellung und -verarbeitung spielen nach wie vor eine Rolle. 1995 registrierte die Berufsgenossenschaft für Nahrungsmittel und Gaststätten (BGN) 1488 Verdachtsfälle einer BK 5101. 1997 wurden noch 1020 Fälle gemeldet. Die BK Haut 5101 nahm den 2. Platz in der Meldestatistik ein [1].

Im Vordergrund stehen ekzematöse Erkrankungen, die sich in der Mehrzahl der Fälle an den Händen manifestieren (Abb.1). Die meisten Beschäftigten zeigen subtoxisch-kumulative Handekzeme, in deren Folge sich eine Kontaktallergie entwickeln kann. Mögliche Konsequenzen sind Arbeitsunfähigkeit und Arbeitslosigkeit. In vielen Fällen besteht der Zwang zur beruflichen Neuorientierung. 


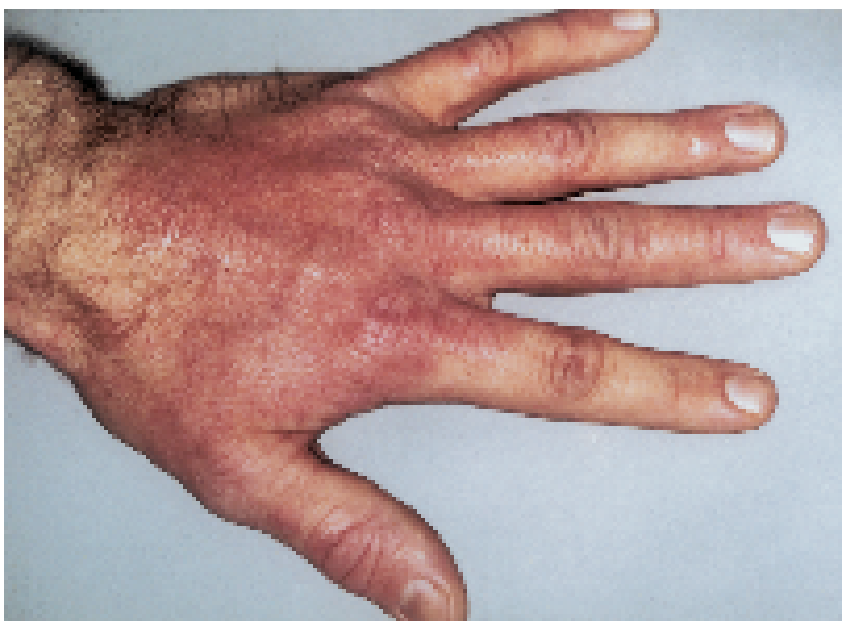

Abb.1 Subakute ekzematöse Hautveränderungen am Handrücken und den Fingerstreckseiten bei einem Bäcker.

Die enormen Kosten für die Umschulungsmaßnahmen und die relativ geringe Aussicht für die Versicherten im neuen Beruf bei der augenblicklich angespannten Arbeitsmarktlage tätig zu werden, unterstreicht eindrucksvoll die Notwendigkeit von effektiven Primär- und Sekundärpräventionsmaßnahmen.

Basierend auf diesen Erkenntnissen wurde 1999 ein Pilotprojekt: „Präventionsprogramm Haut für das Back- sowie Hotel und Gaststättengewerbe“ von der Abteilung für Allergologie und Berufsdermatologie der Klinik für Hautkrankheiten der Friedrich-Schiller-Universität Jena und dem arbeitsmedizinischen Präventionszentrum der Berufsgenossenschaft für Nahrungsmittel und Gaststätten in Erfurt initiiert.

Ziel des Projektes ist es, durch interdisziplinäre Zusammenarbeit die Betreuung von hauterkrankten Arbeitnehmern zu verbessern, die Hauterkrankung zur Abheilung zu bringen und den Versicherten den Verbleib in ihrem erlernten Beruf zu ermöglichen.

Die Betreuung der erkrankten Arbeitnehmer im „Präventionsprogramm Haut für das Back-, sowie Hotel und Gaststättengewerbe" beinhaltet zum einen eine detaillierte Expositionsanalyse durch einen technischen Sachverständigen der BGN, eine ausführliche berufsdermatologische Anamnese und Untersuchung. Auf der Basis der gewonnenen Erkenntnisse wird ein individuelles Hautschutz, -reinigungs und -pflegeprogramm erarbeitet, dem Versicherten erklärt und in übersichtlicher Form schriftlich mitgegeben

Im Einzelnen wird folgendermaßen vorgegangen:

1. Erfassung aller Versicherten mit berufsbezogenen Hauterkrankungen

- Quellen: Hautarztberichte, BK-Meldungen oder Gutachten

- Meldung durch Arbeitnehmer, betreuende Betriebsärzte, andere Präventionsprogramme

2. Auswahl der Versicherten anhand vorliegender Befunde und Unterlagen

3. Information des Versicherten über Programmziele und -inhalte, Einholung der Einverständniserklärungen seitens der Versicherten und Arbeitgeber
4. Expositionsanalyse am individuellen Arbeitsplatz durch den technischen Sachverständigen der BG

5. Vorstellung der Versicherten in der Berufsdermatologischen Sprechstunde an der Klinik für Hautkrankheiten der FSU Jena

- Erhebung der berufsdermatologischen und arbeitsmedizinischen Anamnese

- Reevaluierung und Ergänzung der bisherigen Diagnostik und Therapie

- Erarbeitung eines individuellen Hautschutz, -reinigungs und -pflegeplans unter Einbeziehung der Expositionsanalyse

- Schulung der erkrankten Versicherten

- Bericht an die BG

6. Nachuntersuchungen in 3-6-monatlichen Abständen

- Überprüfung der Effektivität der empfohlenen Präventionsmaßnahmen

7. Hautschutz-Intensivseminare für die erkrankten Versicherten

- Vermittlung von medizinischen Grundlagen

- Vermittlung von technisch-organisatorischen Präventionsmaßnahmen

- Schulung der Anwendung von persönlichen Schutzmaßnahmen (Schutzhandschuhe, Hautschutzsalben)

8. Abschlussbericht an die BG

\section{Ergebnisse}

Gegenwärtiger Stand des Pilotprojekts (Laufzeit 1. 1. 1999-31. 8. 1999)

Seit Januar 1999 wurden 23 Versicherte aus dem Back- sowie Hotel- und Gaststättengewerbe in das Präventionsprojekt eingeschlossen. In der berufsdermatologischen Sprechstunde wurden bisher 16 Versicherte vorgestellt. Davon schieden insgesamt 4 der Versicherten wegen Berufsaufgabe $(n=1)$, befristeten Arbeitsverträgen $(\mathrm{n}=2)$ und aus persönlichen Gründen $(\mathrm{n}=1)$ aus.

Von den bisher untersuchten Versicherten kamen 12 aus dem Backgewerbe und 4 aus dem Hotel- und Gaststättengewerbe (Tab.1).

Tab.1 Gewerbezweigverteilung

\begin{tabular}{|c|c|c|c|c|c|}
\hline \multicolumn{2}{|c|}{ Backgewerbe } & \multicolumn{2}{|c|}{$\begin{array}{l}\text { Hotel- und } \\
\text { Gaststättengewerbe }\end{array}$} & \multicolumn{2}{|c|}{ insgesamt } \\
\hline $\mathrm{m}$ & w & $\mathrm{m}$ & w & $\mathrm{m}$ & w \\
\hline 6 & 6 & 2 & 2 & 8 & 8 \\
\hline \multicolumn{2}{|c|}{12} & \multicolumn{2}{|c|}{4} & \multicolumn{2}{|c|}{16} \\
\hline
\end{tabular}

m: männlich; w: weiblich

Das Durchschnittsalter der Versicherten lag bei 31,3 (Range: 18 - 47) Jahren, Bäcker: 28,5 (Range: 18-47) Jahre, Hotel- u. Gaststätten: 39,8 (Range: 32 - 46) Jahre. Die überwiegende Anzahl der bei uns vorstellig gewordenen Versicherten war in einem Angestelltenverhältnis beschäftigt (Tab. 2).

Bei fast allen Versicherten, die sich in der berufsdermatologischen Sprechstunde im Rahmen des Präventionsprogramms 
Tab. 2 Soziale Stellung im Beruf

\begin{tabular}{llllll}
\hline Selbstständig & \multicolumn{2}{l}{$\begin{array}{l}\text { mithelfendes } \\
\text { Familienmitglied }\end{array}$} & \multicolumn{2}{l}{ Angestellter } \\
\hline Back- & Gastro- & Back- & Gastro- & Back- & Gastro- \\
gewerbe & nomie & gewerbe & nomie & gewerbe & nomie \\
1 & 1 & 2 & 0 & 9 & 3 \\
\hline
\end{tabular}

Tab. 3 Erstdiagnosen und weitere Diagnosen

\begin{tabular}{lc} 
Erstdiagnosen: & Anzahl \\
subtoxisch-kumulatives Handekzem & 12 \\
dyshidrosiformes Handekzem & 1 \\
atopisches Ekzem & 1 \\
Proteinkontaktdermatitis & 1 \\
allergisches Kontaktekzem & 1 \\
gesamt & 16 \\
Zweit- und weitere Diagnosen: & Anzahl \\
subtoxisch-kumulatives Handekzem & 1 \\
Atopie/atopische Diathese & 5 \\
Typ IV-Sensibilisierung gegenüber Berufsstoffen & 4 \\
atopisches Ekzem & 3 \\
dyshidrosiformes Handekzem & 2 \\
gesamt & 15 \\
\hline
\end{tabular}

Tab. 4 Häufigkeit des täglichen Händewaschens

\begin{tabular}{lllll}
\hline $\begin{array}{l}\text { Handwasch- } \\
\text { frequenz }\end{array}$ & $\begin{array}{l}5-10 \times \mid \\
\text { Tag }\end{array}$ & $\begin{array}{l}10-15 \times \mid \\
\text { Tag }\end{array}$ & $\begin{array}{l}15-20 \times \mid \\
\text { Tag }\end{array}$ & $\begin{array}{l}20-25 \times / \\
\text { Tag }\end{array}$ \\
\hline $\mathrm{n}=12$ & 3 & 1 & 3 & 5 \\
\hline
\end{tabular}

vorstellten, lag ein subtoxisch-kumulatives Handekzem $(\mathrm{n}=12)$ vor. Es lagen auch Mischbilder im Sinne einer atopischen Hautdiathese, eines allergischen Kontaktekzems oder atopischen Ekzems vor. Zusätzlich kamen klinisch relevante Typ I-Sensibilisierungen gegen Mehle vor (Tab. 3).

Für die Entstehung des subtoxisch-kumulativen Handekzems konnten wir in den meisten Fällen falsches oder fehlendes Hautschutz- und Hautpflegeverhalten eruieren (Tab. 4, Tab. 5 a u. 5 b), wie z. B. punktuelle und oft falsche Anwendung von Schutzhandschuhen bei stundenlanger Feuchtarbeit, hohe Handwaschfrequenz ( $>15 \times /$ die), aggressive Händereinigung unter Verwendung von heißem Wasser, Bürsten sowie Spülund Reinigungsmitteln als Handreiniger sowie mangelhafte Anwendung von Pflegeprodukten. Akute Exazerbationen allergischer Kontaktekzeme rührten her von ungenügender Aufklärung der Versicherten über Vorkommen der Allergene und unzureichender Meidung der Allergene.

Derzeit ist in 7 (44\%) Fällen eine Verlaufsbeobachtung möglich. Bei einem Versicherten kam es zur Abheilung der Hautveränderungen, bei 2 trat eine Verbesserung ein. 3 Versicherte hatten trotz Verbleib im Beruf einen gleichbleibenden Hautzu-
Tab.5a Hautschutzmaßnahmen

\begin{tabular}{llll}
$\begin{array}{l}\text { Hautschutz- } \\
\text { maßnahmen }\end{array}$ & $\begin{array}{l}\text { Handschuhe } \\
\mathrm{b}=\text { regelmäßig } \\
\text { mäßig } \\
\mathrm{c}=\text { nie }\end{array}$ & $\begin{array}{l}\text { Hautschutzsalbe } \\
\mathrm{a}=\text { regelmäßig } \\
\mathrm{b}=\text { unregelmäßig } \\
\mathrm{c}=\text { nie }\end{array}$ & $\begin{array}{l}\mathrm{a}=\text { regelmäßig } \\
\mathrm{c}=\text { unregelmäßig }\end{array}$ \\
\hline $\mathrm{n}=12$ & $\mathrm{a}=2$ & $\mathrm{a}=1$ & \\
$\mathrm{~b}=3$ & $\mathrm{~b}=0$ & $\mathrm{a}=2$ \\
$\mathrm{c}=7$ & $\mathrm{c}=11$ & $\mathrm{~b}=0$ \\
& $\mathrm{c}=10$
\end{tabular}

Tab. 5b Hautpflege

\begin{tabular}{llll}
\hline Hautpflege & regelmäßig & $\begin{array}{l}\text { unregelmäßig } \\
\text { oder } 1 \times / \text { Tag }\end{array}$ \\
\hline $\mathrm{n}=12$ & 5 & 2 & 5 \\
\hline
\end{tabular}

Tab. 6 Verlauf

\begin{tabular}{llll}
\hline Abheilung & Besserung & Status idem & Verschlechterung \\
\hline $\mathrm{n}=1(14 \%)$ & $\mathrm{n}=2(29 \%)$ & $\mathrm{n}=3(43 \%)$ & $\mathrm{n}=1(14 \%)$ \\
\hline
\end{tabular}

stand. Ein Versicherter zeigte eine Befundprogredienz aufgrund einer Proteinkontaktdermatitis (Tab. 6).

\section{Diskussion und Zusammenfassung}

Subtoxisch-kumulative Handekzeme entwickeln sich durch die chronische Einwirkung unterschwellig hautschädigender Stoffe (Seifen, Desinfektionsmittel, feuchtes Milieu, etc.). Die Summation immer neuer Reizungen führen zur allmählichen Verschlechterung der Barrierefunktion der Epidermis und zur Austrocknung. Die Folgen sind Entzündungserscheinungen der Haut am Einwirkort sowie ein erleichtertes Eindringen von Allergenen und exogenen Noxen. Je nach Konzentration und Einwirkzeit der Irritantien äußern sich Hautdefekte anfangs nur als Minimalbefunde in Form von leichter Rötung, Schuppung sowie kleinen Einrissen im Bereich der Fingerzwischenräume und der Knöchelregion der Handrücken. Diese Anzeichen sind bereits alarmierend und bedürfen einer Optimierung von Hautschutz- und -pflegemaßnahmen, um einer Exazerbation und Chronifizierung des Befundes und der Entstehung von Komplikationen vorzubeugen, wie dem Auftreten allergischer Kontaktekzeme (2-Phasen-Ekzem) [2 - 7].

Effektive Prävention muss, abgestimmt auf die jeweiligen Tätigkeitsprofile und Berufsstoffe am Arbeitsplatz, eine Beratung bez. individueller technisch-organisatorischer Maßnahmen und persönlicher Schutzausrüstung beinhalten (Abb. 2). Zum Schutz vor Irritantien und Kontaktallergenen kommen Einweg- und Mehrweghandschuhe aus dem an die Arbeitsverhältnisse angepassten Materialien sowie Hautschutzsalben zur Anwendung. Um die zusätzliche mechanische und chemische Irritation der Haut beim Händewaschen niedrig zu halten, sollte die Waschfrequenz reduziert, das Reinigungsprodukt (wenn nötig) pH-neutral oder leicht sauer, sowie optimalerweise frei von Farb-, Duft- und Konservierungsstoffen sein. 


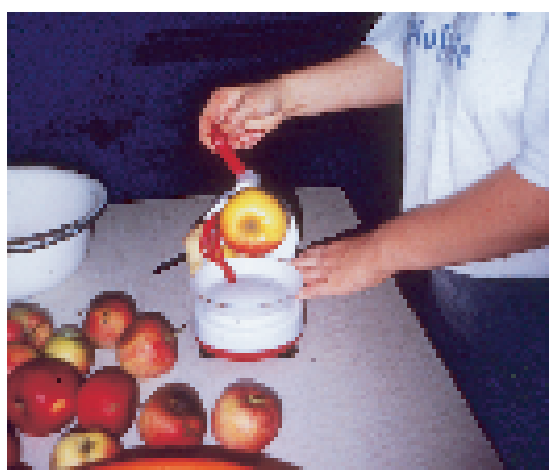

Abb. 2 Teilautomatisierung von Arbeitsgängen.

Bürsten sind in den meisten Fällen zur Händereinigung nicht nötig. Ein gründliches aber sanftes Abtrocknen der Hände, vor allem im Bereich der Fingerzwischenräume mit trockenen Einmalhandtüchern, ist notwendig. Unter Beachtung von Sensibilisierungen und aktuellem Hautzustand sollte die Hautpflege regelmäßig mehrmals am Tag erfolgen, um eine entsprechende Rückfettung zu erreichen [8-12].

Im Rahmen des „Präventionsprogramms Haut für das Backsowie Hotel- und Gaststättengewerbe“ konnten wir in 3 von 7 Fällen ( $43 \%$ ) bereits eine Besserung des Hautbefundes erzielen, in weiteren 3 Fällen (43\%) war der Befund trotz fortgeführter Berufstätigkeit konstant. Ein Versicherter zeigte eine Befundprogredienz aufgrund einer Proteinkontaktdermatitis. Da effektive Schutzmaßnahmen nicht realisierbar waren, wurde dem Versicherten ein Berufswechsel empfohlen. Alle Versicherten des Präventionsprogramms „Haut“ hatten sich bereits in hautfachärztlicher Behandlung befunden. In den meisten Fällen war es darunter zu keiner stabilen Besserung des Hautzustandes gekommen. Wer Schutzmaßnahmen am Arbeitsplatz durchführte, hatte dies fast immer selbst finanziert. Eine gründliche Beratung und Aufklärung diesbezüglich war nicht erfolgt.

Die Ergebnisse zeigen die Notwendigkeit und die ersten Erfolge von effektiven Sekundärpräventionsmaßnahmen.

\section{Literatur}

${ }^{1}$ Jahresbericht „BGN in Zahlen“. Editors BGN. B. C. Verlag \& Mediengesellschaft, 1996

${ }^{2}$ Brasch L, Burgard J, Sterry W. Common pathogenetic pathways in allergic and irritant contact dermatitis. J Invest Dermatol 1992; 98: $166-170$

${ }^{3}$ Wüthrich B. Zur Genese des Bäckerekzems. Hautarzt 1970; 221: 214-218

${ }^{4}$ De Jong O. Etiology of dermatitis in bakers. Lancet 1923; 1: 894

${ }^{5}$ Bauer A, Bartsch R, Stadeler M, Schneider W, Grieshaber R, Wollina U, Gebhardt M. Development of occupational skin diseases during vocational training in baker and confectioner apprentices: a follow-up study. Contact Dermatitis 1998; 39: 307 - 311

${ }^{6}$ Bauer A, Seidel A, Bartsch R, Wollina U, Gebhardt M, Diepgen TL. Entwicklung von Hautproblemen bei Berufsanfängern in Hautrisikoberufen. Allergologie 1997; 20: 179-183

${ }^{7}$ Peltonen L, Wickström G, Vaahtoranta M. Occupational dermatoses in the food industry. Dermatosen 1985; 33: 166-169

${ }^{8}$ Diepgen TL. Adäquater Hautschutz - Möglichkeiten, Grenzen, Gefahren. Allergologie 1993; 16: 25 - 34

${ }^{9}$ Mathias CG. Prevention of occupational contact dermatitis. J Am Acad Dermatol 1990; 23: $742-748$

${ }^{10}$ Halkier-Sorensen L, Thestrup-Pedersen K. The efficacy of a moisturizer (locobase) among cleaners and kitchen assistants during everyday exposure to water and detergents. Contact Dermatitis 1993; 29: $266-271$

${ }^{11}$ Wigger Alberti W, Maraffio B, Wernli M, Elsner P. Training workers at risk for occupational contact dermatitis in the application of protective creams: efficacy of a fluorescence technique. Dermatolgy 1997; 195: 129-133

12 Wigger-Alberti W, Elsner P. Preventive measures in contact dermatitis. Clin Dermatol 1997; 15: 661 -665

\section{Dr. med. Andrea Bauer}

Klinik für Hautkrankheiten

Friedrich-Schiller-Universität Jena

Erfurter Straße 35

07740 Jena 